\title{
The Decision Aid Leak Notification System for Pigging False Alarm
}

\author{
Thanet Vilasmongkolchai ${ }^{1}$ and Thichakorn Visansakon ${ }^{2}$ \\ ${ }^{1}$ Aviation Maintenance Program, Faculty of Technology, Siam Technology College, Thailand. \\ ${ }^{2}$ International Business Management, International College, Siam Technology College, Thailand.
}

\begin{abstract}
The pipeline inspection using dynamics pressure has been continuous development with the advantages on low noise, long range, accessible, and no electrical spark occurred. However, the leak indication signal seems to positive false alarm because of sensor's sensitivity. The pigging signal is the one of most miss interpretation and annoy on-site staff and system administrator. The signal of leak simulation and pigging has been collected and study of their characterization difference, then identified as scrutinize condition. This research compared the proposing method to the conventional (with and without decision aid technique). On the results, the advantage of decision aid technique was reduce false alarm during pigging process up to 87\% for highest accuracy.
\end{abstract}

Keywords: Dynamics Pressure, Decision aid, Pipeline, Leakage, Pigging.

\section{Introduction}

The use of pipeline as fuel transmission method is the most convenient, fast, and safe. Nevertheless, the pipeline network was constructed underground and the deteriorated did not easy to observe. The lacking of schedule inspection and lifetime prediction should make a harmful accident which affected to environment, life, and asset. Online monitoring system has been proposed for the first decision aid such as automatic shut off the main valve and given a notification to confederate. However the SCADA has been designed to support this incident, but the function will work only the instant change of pressure or the large pressure drop and unable to detect a small leakage and cannot locate the leak location accurately. There are some of non-destructive testing technique capable to solve this problem, the small leakage was detect instantaneously and able to specify the leak location. As the previous literature, the effective and popular used the online pipe leak detection system is the acoustic emission method (AE) and dynamics pressure method (DP). These method capable to use with the pre-installed pipeline and safe from the explosion cause.

The leak occurred produce acoustic signal and propagating along the pipeline to the sensor, with the using principle of acoustic and simple law of motion, the leak location has been calculated. Unfortunately, the acoustic emission method was inappropriate to use with the long pipeline application. Because, the highly attenuation of acoustic signal, AE sensor need to install too many points through pipeline length. The dynamics pressure method was outstanding cause of the signal propagated in fluid instead of pipe wall. So, the long distance leak detection was capable also in easy installation [1-4]. For the leak location processing, inter-station communication was necessary, with the timing parameter and sensor installation location as minimum information to locate leak position. Unfortunately, during pipeline cleaning process and internal pipeline inspection by pigging also generate signal like the leak indication signal and make false alarm. From this 
concerning, the signal processing technique and heuristic condition has presented for decision aid of leak notification system.

\section{Research Background}

\subsection{Dynamics Pressure Transducer}

Dynamic pressure transducer is the pressure measurement device that measuring pressure transients or instantaneous change of pressure in fluid. The fast response with micro-second rise time fetch the bandwidth of transducer response in range of $\mathrm{kHz}$ and over, so it capable to detection of leak in pipeline. When leak occurred, the pressure instant change and propagate as wave through fluid to the transducer. The advantages in low interference, low signal attenuation, and easy installation make the dynamics pressure transducer popular in leak monitoring system.

\subsection{Leak Locationing}

The location of the leak was calculated based on the velocity of pressure wave that propagate in the fluid, by examining the difference between indication signal's arrival times $(\Delta t)$ at the two opposing transducers $\left(S_{1}\right.$ and $S_{2}$, and the length of the pipeline segment, as shown in the equation below

$$
\mathrm{X}=(\mathrm{L}-\Delta \mathrm{t} \cdot \mathrm{v}) / 2
$$

$$
\begin{aligned}
& \text { where } \quad \mathrm{x} \quad \text { is the distance between the first sensor and the leak point }(\mathrm{m} \text {.) } \\
& \mathrm{L} \quad \text { is the distance between sensors installation ( } \mathrm{m} \text {.) } \\
& \Delta \mathrm{t} \quad \text { is the time difference of the two sensors captured leak indication signal (s.) } \\
& \mathrm{v} \quad \text { is the velocity of negative pressure wave propagation }(\mathrm{m} / \mathrm{s} \text {.). }
\end{aligned}
$$

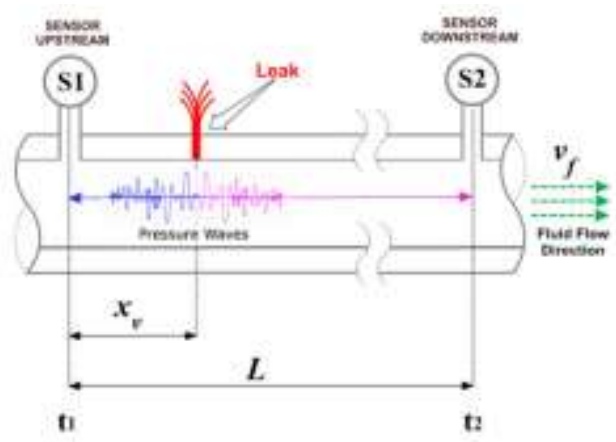

Fig. 1: pipe leak locationing using negative pressure wave method [5]

\subsection{Pigging}

Pigging in the process of using devices (pigs) to perform various maintenance operations inside the pipeline. Pigs are also used to Pigs may be used in internal cleaning, internal coating, liquid management, batching, and inspection. The pigging process usually do not interrupt production, though some product can be lost when the pig is extracted [6]. The traveling of pigs through the pipe usually abrasive and impact the pipe wall and gave the transient of pressure in media which might misinterpretation as leakage occurred.

\section{Experimental Setup}

The diagram below can divided into two parts, leak indicator sensing and signal processing. The part of leak indicator sensing consist of dynamic pressure transducer and signal improvement circuit. For the signal processing part, data acquisition card connected to the main processing unit and use the main processing unit's time base as a reference in leak location computation. On the experiment, the two transducers was set at 75 kilometers distance along natural gas pipeline with operation pressure 35 bar, the leakage would simulate by 
bleed valve setup at the operation center (OC), and the pigs would run along this pipeline with 180 kilometers distance from the first transducer. Firstly, the signal of leak simulation and pigging would collect in-situ for analyze the difference in signal characteristic. Then, the total collected data will retest with the designed decision aid algorithm for find out the accuracy in leak notification.

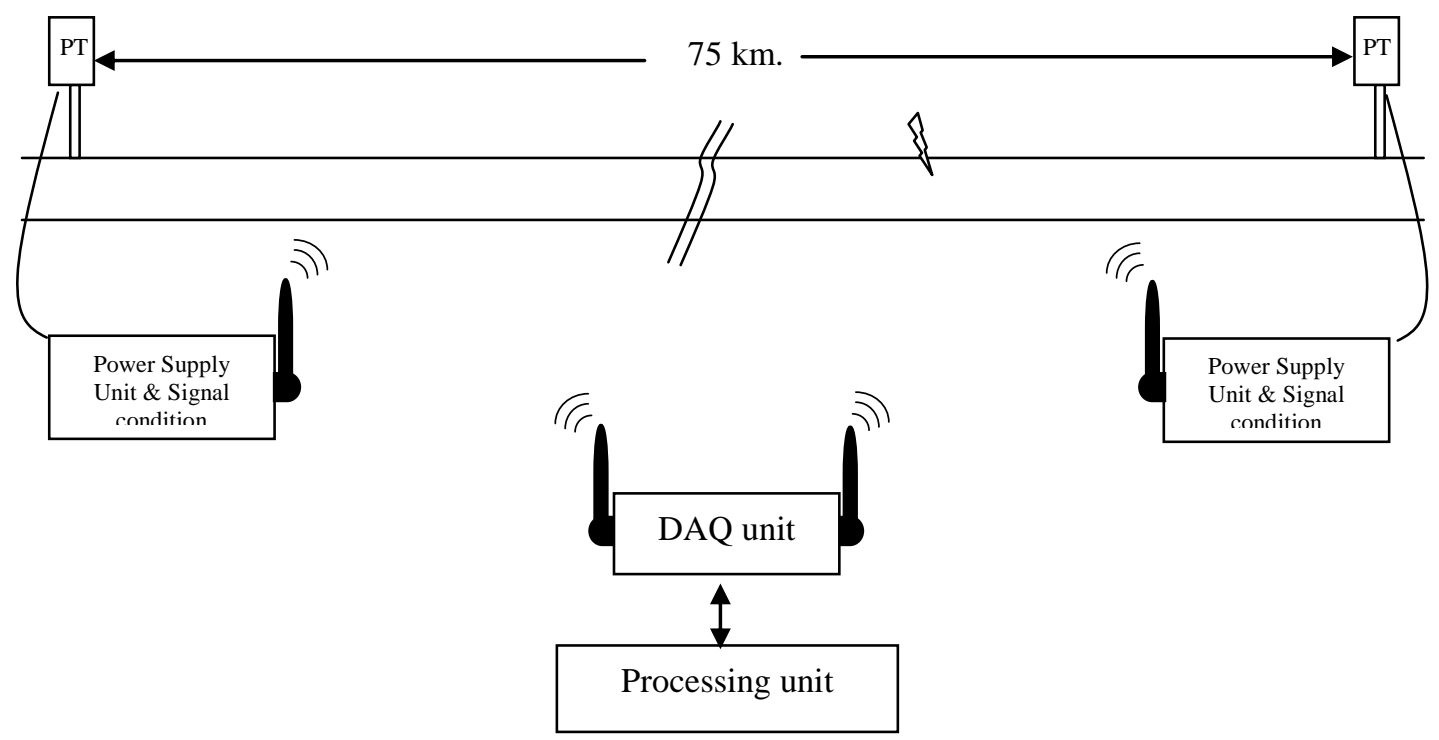

Fig. 2: overall of the system

\subsection{Characteristic of Signals}
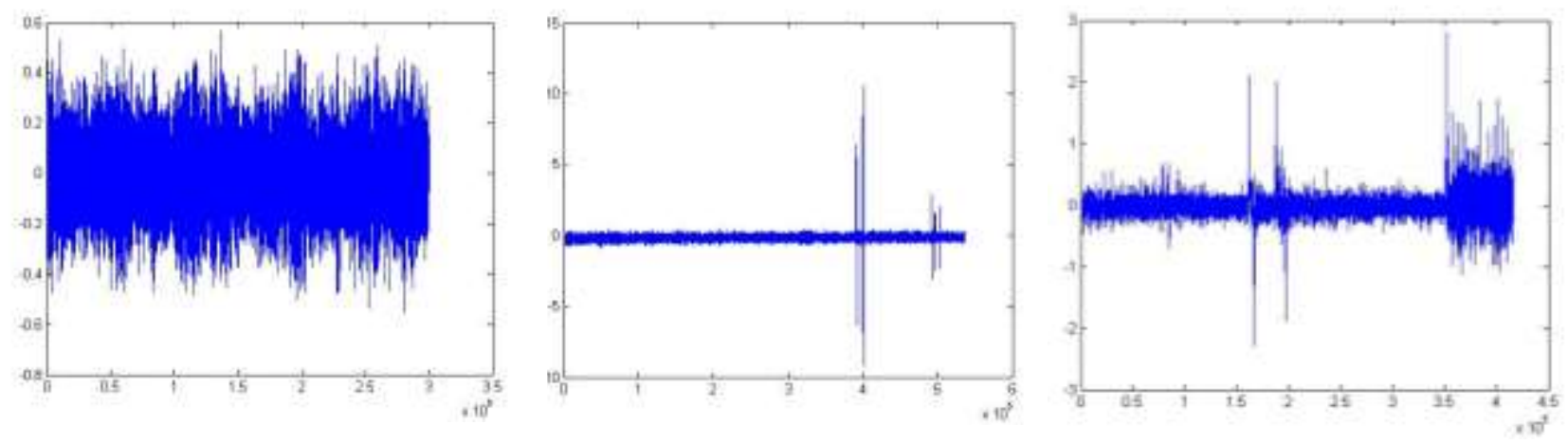

Fig. 3: background signal (a), the leak simulation signal (b), and the pligging signal (c)

The figures above presented the 3 appearances of recording signal (not related in same scale). Background signal demonstrated to a random noise from the system without pressure instantly change. Leak simulation signal is the sharp spike over the background level. Pigging signal also indicated the sharp spike as the leak simulation and some captured signal display as higher amplitude of the background signal. The Fig. $3 \mathrm{c}$ is the pigging signal that first captured after pigs launce with estimated distance 100 kilometers from the transducer. So, it seems difficult to identify the leak signal and make system notified frequently especially in maintenance period.

\subsection{Post-Processing}

When the leak has been simulated during pig running (Figure 4), it was ambiguous appearance signal and cannot identify that the pipe had leaked or not. From the collected information, the observing only in time domain is not enough to decide to automatically shut off the block valve. So, the frequency response in each captured signal was present in comparison as the figure 5 . The frequency at the range between $5 \mathrm{kHz}$ to $15 \mathrm{kHz}$ 
was the different of leak simulation signal. So, the use of electronics filter or digital filter is the most suitable for the first step screening.

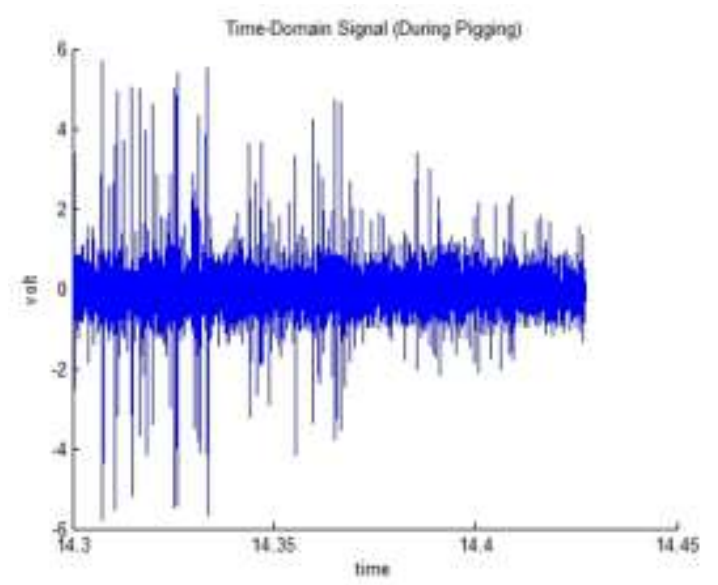

Fig. 4: the signal of leak simulation during pigging process

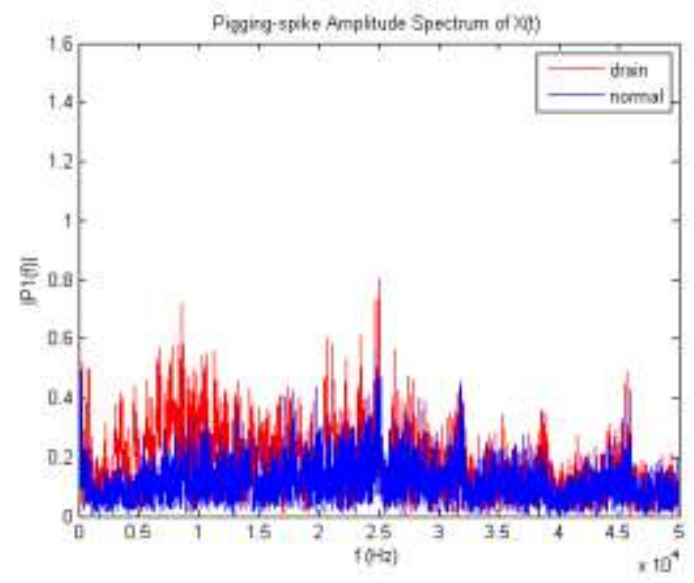

Fig. 5: comparison between normal pigs running (blue) and leak simulation during pigging (red)

Threshold is the commonly method to indicate the leak signal, the signal that has amplitude higher than the background signal level could preliminary designate as leak indicator signal, but only amplitude pursuit was insufficient. Hence, this study propose the post-processing procedure for higher accuracy leak detection as follows;

- Band-Pass Filter: the signal was considered only in range of $5 \mathrm{kHz}-15 \mathrm{kHz}$ frequency,

- Amplitude Screening: the signal that has three or greater signal to noise ratio was set to leak indicator,

- Leak Indicator Capture: leak indicator signal would be capture in one second window, then

- Repeat Parameter Checking: for frequency analysis.

Through this procedure, if the leak indicator signal has been captured more than two times within five seconds, so the leak indication can be confirmed.

\section{Results and Discussion}

From the experiment, time domain data had collected in-situ for leak exploration. The leak was simulated by the using of bleed valve and vent to atmosphere both in operation and pigging situation. The time series data was imported to the decision aid system and find out the percentage of accuracy in signal identification and the results had shown in the table. 
TABLE I: The Accuracy of Signal Identification

\begin{tabular}{lccc}
\hline \hline \multirow{2}{*}{ Type of Operation } & \multicolumn{3}{c}{$\%$ of accuracy } \\
\cline { 2 - 4 } & Amplitude Method & Frequency Method & Decision Aid System \\
\hline normal gas flow & 96 & 100 & 100 \\
leak simulation & 72 & 87 & 96 \\
pigging & 43 & 69 & 82 \\
leak simulation \& pigging & $\mathrm{n} / \mathrm{a}$ & 74 & 87 \\
\hline \hline
\end{tabular}

Although the amplitude method (threshold) and frequency method were acceptable in identify leak indication from normal gas flow situation, but cannot specify the type of operation in accurate especially during pigging process. The decision aid system is the combination method of signal processing technique and shown the capability in signal identification. On the leak simulation during pigging, the propose method achieved the 87 percent identification accuracy. For the further study, the decision aid system need to develop by accuracy concerning and processing time for the appropriate in practical.

\section{References}

[1] Y. Zhang, et. al, "Leak detection monitoring system of long distance oil pipeline based on dynamic pressure transmitter," Measurement, 2014, Vol. 49, pp. 382-389.

[2] H. Jin, et. al., "Integrated leakage detection and localization model for gas pipelines based on the acoustic wave method," Journal of Loss Prevention in the Process Industries, 2014, Vol. 27, pp. 74-88.

[3] LB defence Group, Wavealert: Acoustic Leak Detection System for pipeline, 2007.

[4] W. Lin and X. Zhang, "A Novel Approach for Dynamic Pressure Transducer Based Pipeline Leak Detection," Proceedings of the 6th World Congress on Intelligent Control and Automation, Dalian China, 21 - 23 June 2006.

[5] PIPE-TECH. (2014). Operating Principles of Negative-pressure Wave Acoustic Leak Detection System. Pipeline Technologies and Services. [Online]. Available: http://www.pipetechs.com/?page_id=539

[6] Society of Petroleum Engineers, (2015), Pipeline Pigging, PetroWiki, [Online]. Available: http://petrowiki.org/Pipeline_pigging 